\title{
Does regulating the sale of high-strength beer and cider impact hospital admissions with decompensated alcohol- related liver disease: A retrospective cohort study
}

\author{
Authors: Yazan Haddadin, ${ }^{\mathrm{A}}$ Dev Katarey, ${ }^{\mathrm{B}}$ Manavi Sachdeva, ${ }^{\mathrm{C}}$ Laura Vickers, ${ }^{\mathrm{C}}$ Ishleen Kaur, ${ }^{\mathrm{C}}$ Ahmed Hashim ${ }^{\mathrm{B}}$ and \\ Sumita Verma ${ }^{\mathrm{D}}$
}

\begin{abstract}
Objective
'Sensible on Strength' (SoS), a local public health initiative, is aimed at reducing high-strength beer and cider availability. The objective of this study was to assess its impact on local hospital admissions with alcohol-related liver disease (ALD) and on drinking behaviour.
\end{abstract}

\section{Design}

This was a retrospective cohort study in patients admitted with decompensated ALD, 3 years before and 3 years after the introduction of the SoS initiative.

Hospital records of 143 index admissions with

decompensated ALD were reviewed. Outcomes measures were the severity of liver disease on admission and mortality (inpatient and long-term), and change (if any) in alcohol drinking behaviour.

Results

Comparing patients admitted in both phases, there were no significant differences in liver prognostic scores, liverrelated complications, length of stay and inpatient/long-term mortality $(p>0.05)$. However, the SoS initiative was associated with a $33 \%$ move away from beer and cider consumption ( $36.3 \%$ vs $54.0 \% ; p=0.034$ ), but without a significant change in units of alcohol consumed. The Model for End-stage Liver Disease (MELD) score was the only independent predictor of inpatient mortality (odds ratio $1.25 ; p=0.025$ ).

Conclusion

Despite having no apparent impact on the clinical spectrum of local ALD admissions, it is conceivable that longer follow-up is needed to determine the true impact of this initiative.

Authors: ${ }^{\text {A }}$ core medical trainee, Brighton and Sussex University Hospitals NHS Trust, Brighton, UK; ${ }^{\mathrm{B}}$ academic specialist trainee, Brighton and Sussex University Hospitals NHS Trust, Brighton, UK and Brighton and Sussex Medical School, Brighton, UK; ${ }^{C_{\text {foundation }}}$ year trainee, Brighton and Sussex University Hospitals NHS Trust,

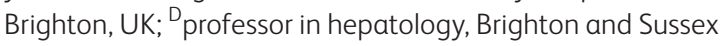
University Hospitals NHS Trust, Brighton, UK
KEYWORDS: Alcohol, cirrhosis, public health, alcohol-related liver disease, regulations

DOI: 10.7861/clinmed.2019-0390

\section{Introduction}

Alcohol misuse continues to be one of the leading causes of liver disease in the UK, with over one-third of a million hospital admissions in England between 2016 and 2017 due to an alcoholrelated primary condition. Moreover, alcohol-specific mortality is also on the rise, with 5,507 directly attributable deaths in England alone in 2016 (up by $11 \%$ compared with 2006). ${ }^{1}$

In addition to the human toll, alcohol has a huge societal impact. A report published in 2016 estimates the annual cost of alcohol to be between $1.3 \%$ and $2.7 \%$ of gross domestic product. $^{2}$

The burden of alcohol-related liver disease (ALD), however, is not shared equally between different regions of the UK or, indeed, different socioeconomic groups. Public Health England (PHE) have identified certain high-risk areas for ALD. Coastal towns, where night-time drinking plays a big part in the economy, and deprived areas, with high prevalence of harmful drug use, seem to be particularly susceptible.

Brighton and Hove in the UK is home to the most populous seaside community in England and Wales according to the 2011 census. $^{3}$ A city with a population of almost 300,000 people, two universities and a large lesbian, gay, bisexual and transgender community, its economy relies heavily on tourism and entertainment, of which night-time drinking plays a large part. There is no surprise, therefore, that locally they have one of the highest rates of alcohol-specific mortality (16.1/100,000; national average $10.6 / 100,000$ ) and number of admission episodes for alcohol-specific conditions (697/100,000; national average 570/100,000) in south-east England. ${ }^{4}$

As highlighted by the recent Lancet report on liver disease, strong cheap alcohol plays a particularly damaging role, a fact acknowledged by government legislators.

Scotland has been a major lead in the UK with the introduction of a national minimum unit pricing in May 2018. But local 
strategies could have the potential to expand the benefits seen with national initiatives by tackling the availability and marketing of cheap high-strength beverages. A report suggests that as high-strength alcohols have become more affordable since 1980, alcohol-related deaths in England and Wales have more than trebled. $^{5}$

In November 2013, the Brighton and Hove local city council launched a voluntary 'Sensible on Strength' (SoS) campaign to encourage off-licences against selling high-strength beer, lager or cider (above $6 \%$ alcohol by volume) and implement other good practices including a strict refusal policy, round-the-clock closed-circuit television monitoring and displaying of an SoS sticker. Two years following the initiative, there has been a 31\% drop in street drinking. Other cities (Manchester) adopting similar initiatives ('Reduce the Strength') also observed a fall in alcoholrelated crime in intervention compared to control areas that continued to sell cheap high-strength beverages ( $41 \%$ vs $15 \%$, respectively). ${ }^{2}$

The aim of this study was to assess the impact of the SOS initiative on local hospital admissions with ALD as well as on alcohol drinking behaviour in this subgroup of patients.

\section{Patients and methods}

This retrospective cohort study was conducted at a large teaching hospital in south-east England from 3 years before (phase 1) to 3 years after (phase 2) the launch of the SoS initiative (November 2010 to November 2016). Patients with a primary coded diagnosis of ALD (all ' $K 70$ ' International classification of diseases, 10th revision (ICD-10) codes) during the study period were identified from the hospital's database. ${ }^{6}$ Their medical and electronic records were then retrospectively reviewed to identify those with an index presentation with decompensated cirrhosis.

Decompensated cirrhosis was defined by the presence of one or more of the following on admission: variceal bleeding, ascites, hepatic encephalopathy (HE) and jaundice, with an admission Child-Pugh score (CPS) of $\geq 7$. $^{7}$ CPS can be difficult to determine retrospectively as it includes subjective assessment of ascites and HE. Severity of ascites and HE were based on a senior clinician's review as documented in the medical records and, if needed, supported by radiological data. Most individuals with HE fulfilled study inclusion criteria, irrespective of scores allocated for HE. We selected decompensated cirrhosis as an eligibility criterion to ensure that the hospital admissions were directly related to the liver disease.

While there are now accepted clinical criteria for the diagnosis of alcoholic hepatitis, unfortunately about $50 \%$ of our cohort did not have an aspartate transaminase (AST) performed as only the alanine transaminase (ALT) is included in the standard liver panel at our hospital. ${ }^{8}$ We therefore did not stratify patients based on absence or presence of alcoholic hepatitis.

Our outcome measures were to assess impact of SoS initiative on:

$>$ the severity of liver disease at index presentation with decompensated ALD as assessed by liver prognostic scores (CPS, Model for End-stage Liver Disease (MELD) score and UK Model for End-stage Liver Disease (UKELD) score), length of hospital stay, and inpatient and long-term mortality

> change (if any) in alcohol drinking behaviour in our patient group. $^{9,10}$
ALD was defined as long-standing alcohol excess ( $>14$ units / $>112 \mathrm{~g}$ in 1 week regardless of gender) and/or if medical records stated alcohol as the aetiology of liver disease. ${ }^{11}$

\section{Data collection}

Alcohol history was obtained from the medical records. Our hospital admission pro forma has a specific section for this. We recorded data on duration, nature and amount of alcohol being consumed. If there was a discrepancy in this data between the admitting medical and gastroenterology/hepatology team, the latter's version was recorded. We also collected data on patients who, as a result of feeling unwell, reduced their alcohol intake to the point of abstinence prior to admission. If a patient expressed a preference to more than one type of alcohol, this was also noted.

In addition to the alcohol history and outcome measures mentioned, the following data were retrospectively collected from medical and electronic records: patient demographics, comorbidities, presence of ascites, infections, acute kidney injury (AKI), HE, portal hypertension-related gastrointestinal (GI) haemorrhage, and the number and duration of subsequent hospital admissions.

Overall survival was calculated from the date of hospitalisation until May 2018 and censored at death (based on hospital electronic records). Cause of death was obtained largely from hospital records and inpatient death certificates. Deaths arising from hepatocellular carcinoma (HCC), liver failure and portal hypertension-related GI bleeding were defined to constitute liver-related mortality. Patients with any other form of coexistent chronic liver disease (viral hepatitis, iron overload, and biliary or autoimmune disease) were excluded.

There are approximately 323 premises that sell alcohol in the city. Table 1 shows the timeline of those premises that joined the SoS scheme from 2013 to the time of writing. The campaign therefore saw the participation of only 154 (41\%) premises during our study period, although this had increased to $74 \%$ by 2019. ${ }^{12}$

Table 1. Number of premises signed up to the Sensible on Strength scheme by year

\begin{tabular}{llll} 
Year & $\begin{array}{l}\text { Official } \\
\text { members } \\
\text { of the SoS } \\
\text { initiative, } \mathbf{n}\end{array}$ & $\begin{array}{l}\text { Unofficial } \\
\text { members } \\
\text { of the SoS } \\
\text { initiative also } \\
\text { banning cheap } \\
\text { high-strength } \\
\text { drinks, } \mathbf{n}\end{array}$ & $\begin{array}{l}\text { Total } \\
\text { (percentage of } \\
\text { all premises), } \\
\mathbf{n}(\%)\end{array}$ \\
2013-14 & 79 & 0 & $79(24.5)$ \\
$2014-15$ & 119 & 0 & $119(36.8)$ \\
$2015-16$ & 131 & 23 & $154(47.7)$ \\
$2016-17$ & 159 & 39 & $198(61.3)$ \\
$2018-19$ & 178 & 48 & $226(70.0)$ \\
$2019-20$ & 191 & 49 & $240(74.3)$ \\
\hline SoS $=$ Sensible on Strength. & &
\end{tabular}




\section{Statistical analysis}

Data are presented as mean ( \pm standard deviation), median (interquartile range) or number (\%) and all reported $p$ values are two-tailed. The Mann-Whitney $U$ test and Student's t-test were used to compare non-parametric and parametric continuous variables, respectively, and categorical data were compared using the $\chi^{2}$ test. Kaplan-Meier (KM) survival tables and curves were generated and factors compared using the logrank test. Binary logistic regression analysis was performed to identify factors associated with in-hospital mortality. Parameters with $p$ values $<0.10$ in univariate analysis were selected for inclusion in a multivariable regression model to determine independent predictors of in-hospital mortality. Survival analyses were performed in the whole cohort and statistical analyses were undertaken using SPSS Version 25.0 (IBM Corp, Armonk, USA).

This study was classified as service evaluation by our institute's research and development department and formal ethics approval was not deemed necessary.

\section{Results}

During the study period, we identified 612 individual patients with an ALD coded admission who lived within the SoS initiative's catchment area. Of these, we excluded 462: incorrectly coded as having chronic liver disease or the extent of liver disease was not significant enough to meet our strict inclusion criteria $(n=338)$; index admission was outside our study period $(n=63)$; presence of coexisting liver disease (predominantly viral hepatitis; $n=61$ ); and missing notes $(n=7)$. One-hundred and forty-three individuals with an index presentation with decompensated ALD during the study period were therefore eligible for inclusion in the study.

Supplementary material S1 shows baseline clinical and demographic data in the whole cohort, and the two phases. Overall, at admission, five (3.5\%) had CPS-A disease, 52 (36.4\%) had CPS-B and 86 (60.1\%) had CPS-C disease, with 75 (52.4\%) having a MELD score $>20$. Seventeen $(11.9 \%)$ were aged $<40$ years and $24(16.8 \%)$ were aged $>60$ years. Comparing admissions in phase 1 and 2 there were no significant differences in patient demographics, liver prognostic scores, prevalence of cirrhosis-related complications and length of hospital stay.

Data on alcohol units consumed per day were available in 139 $(97.2 \%)$ patients, duration of alcohol use in 78 (54.5\%) patients and nature of alcohol use in 129 (90.2\%) patients.

Overall, consumption of beer and cider were significantly lower in phase 2 compared to phase 1 (36.3\% vs $54.0 \%$, respectively; $p=0.034$; Fig 1a). Over the same time period, there was a slight increase in wine and spirits consumption, although this was not significant $(60.0 \%$ vs $52.4 \%$, respectively; $p=0.361)$. The percentage with missing data on the type of alcohol consumed were not significantly different during phase 1 and 2 (7.9\% vs $11.3 \%$, respectively; $p=0.503$ ).

Fig $1 \mathrm{~b}$ shows the yearly trends in nature of alcohol consumption (beer and cider vs wine and spirits) following the introduction of the SoS initiative.

\section{Mortality}

Overall mortality in the whole cohort (as of May 2018) was 75 $(52.4 \%)$ of whom 26 (34.7\%) died during the index hospital

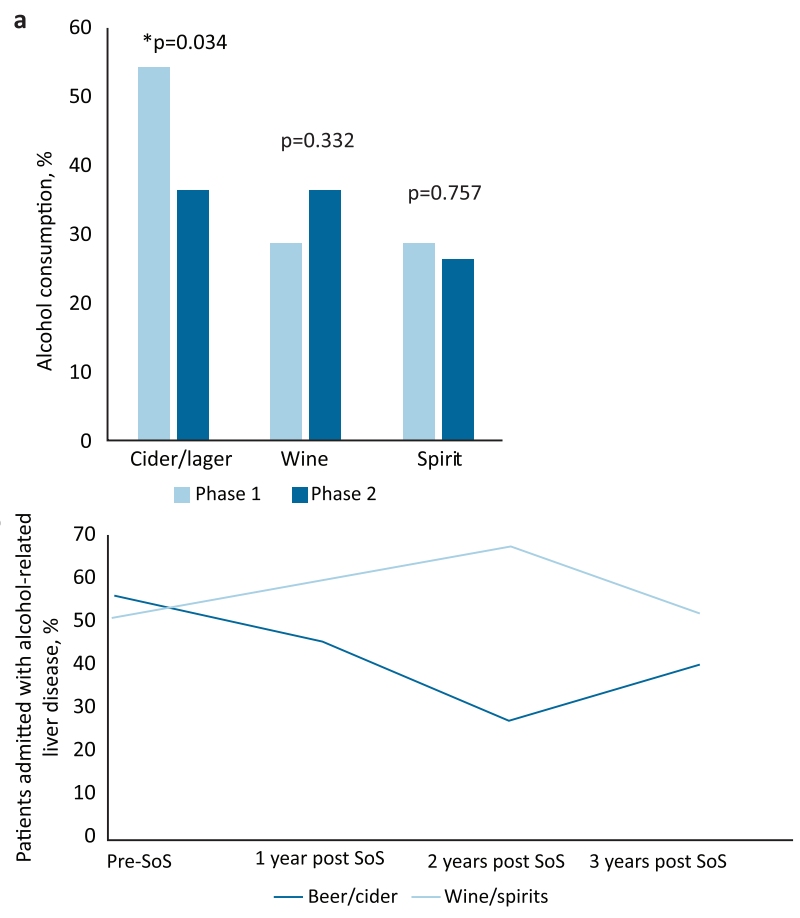

Fig 1. a) Prevalence of consumption of different types of alcohol in phases 1 and $2{ }^{*}{ }^{*}=$ statistically significant, $p<0.05$. b) Yearly trend in the choice of alcohol (as a percentage of patients admitted with alcoholrelated liver disease) following introduction of Sensible on Strength initiative. SoS = Sensible on Strength.

admission (the cause of death being liver-related in all of these cases). There was no difference in inpatient mortality between phase 1 and phase $2(19.0 \%$ vs $17.5 \%$, respectively; $\mathrm{p}=0.812$ )

Of the 49 who died after their index hospitalisation, this was liver-related in $25(51 \%)$. In the remaining 24 patients, the cause of death was unknown: we were unable to access the primary healthcare records in 20 and four were referred to the coroner without an available outcome.

See supplementary material S2 for univariate analysis of baseline demographic and clinical variables associated with inpatient mortality during index hospitalisation. There was multicollinearity between creatinine, AKI, bilirubin, HE, international normalised ratio and the CPS and also between sodium, MELD and UKELD scores. Therefore, only the following variables were entered into multivariate regression analysis: age, gender, duration of alcohol use, CPS, MELD, infection rates and ICU stay. The only independent predictor of mortality during index hospitalisation was the MELD score (odds ratio 1.25; $95 \%$ confidence interval 1.03-1.51; $p=0.025$; Table 2).

Fig 2 shows the KM analysis of overall survival for patients admitted in phase 1 and 2 . There were no statistically significant differences in the two cohorts $(p=0.396)$.

\section{Discussion}

This is one of the few published studies in England that attempts to assess whether local regulations of alcohol sales influence drinking behaviour and hospital admissions in patients with ALD. 
Table 2. Multivariate analysis of baseline

demographic and clinical variables associated with

inpatient mortality following index hospitalisation

with decompensated alcohol-related liver disease

\begin{tabular}{llll} 
& $\begin{array}{l}\text { Odds } \\
\text { ratio }\end{array}$ & $\begin{array}{l}\mathbf{9 5 \%} \\
\text { confidence } \\
\text { interval }\end{array}$ & p value \\
Age, years & 1.09 & $0.97-1.22$ & 0.140 \\
Gender, male & 3.33 & $0.17-67.5$ & 0.433 \\
Duration of alcohol & 1.04 & $0.97-1.12$ & 0.336 \\
intake, years & & & \\
Child-Pugh score & 0.64 & $0.31-1.31$ & 0.224 \\
MELD score & 1.25 & $1.03-1.51$ & $\mathbf{0 . 0 2 5}$ \\
Presence of infection & 2.68 & $0.27-26.2$ & 0.397 \\
ICU stay, days & 4.58 & $0.17-126$ & 0.369 \\
\hline ICU = intensive care unit: MELD & Model for End-stage Liver Disease.
\end{tabular}

ICU = intensive care unit; MELD = Model for End-stage Liver Disease

Though there was a 33\% reduction in the consumption of beer and cider post introduction of the SOS initiative, the total number of alcohol units consumed did not change. This might explain why the SOS initiative did not impact the number and severity of hospital admissions with ALD nor have an effect on ALD-related mortality. In fact, the MELD score remained the only independent predictor of inpatient mortality.

Our data lends credence to the existing body of evidence that interventions at a population (albeit local) level can modify alcohol drinking behaviour. ${ }^{5,13}$ This is despite the impact of SoS being limited to the type of alcohol rather than the total amount of alcohol consumed; the impact of the type of alcohol consumed on ALD outcomes, in fact, remains controversial.

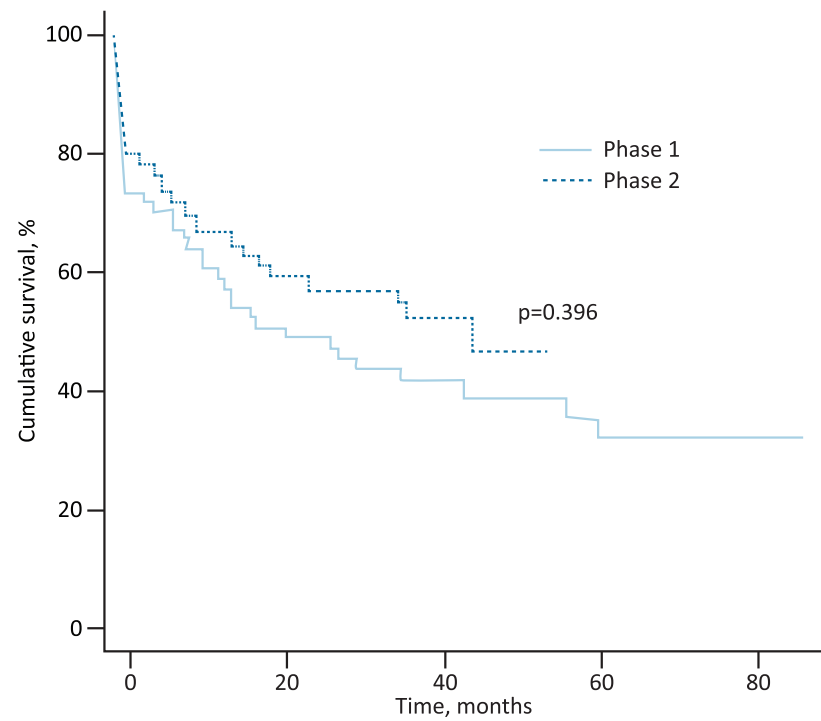

Fig 2. Kaplan-Meier graph showing overall mortality during phase 1 and 2 admissions.
A large longitudinal study in the USA, where researchers collected data on type of alcohol sales in 50 states over 12 years, found that, after controlling for other covariates, every percentage increase in spirit sales was associated with a significant increase in cirrhosis mortality rates, no such effect being observed for beer or cider. ${ }^{14}$

Additionally, in Sweden, over two separate time periods, reduction in mortality from liver disease between 1969 and 2001 seems to closely parallel downward trends in sales of spirits but not that of beer or wine. ${ }^{15}$

In contrast, data from Iceland that spans almost 3 decades showed the importance of total units of alcohol consumed, rather than the type of alcohol, as the main determinant of cirrhosis mortality. A lift of the ban on beer sales in 1989 was followed by a $30 \%$ rise in alcohol intake, this specifically being driven by an increase in beer consumption. A drop in the intake of spirits over this time period did not prevent the increase in recorded cirrhosis mortality in men. ${ }^{16}$ This corroborates our data suggesting that it is the amount rather than the nature of alcohol that determines ALD outcomes.

Although outcomes in ALD are gender dependent, we were unable to assess this due to the small number of women in our cohort. ${ }^{17}$ However, there was no difference in the gender distribution between phase 1 and phase $2(p=0.835)$.

Our study focused on the impact of SOS on ALD and drinking behaviour, but it is important to be cognisant of the fact that moving an already financially deprived cohort away from cheap beverages to more expensive drinks could potentially have negative social consequences. This needs to be carefully addressed in the future when the full impact of the SoS initiative is more apparent.

Our study did have limitations. The voluntary nature of this public health campaign meant that patients could access high-strength drinks from outlets that had not signed up to the SoS scheme, which amounted to more than $50 \%$ of premises during our study period. Secondly, reliably estimating alcohol intake retrospectively is fraught with difficulty. As with national health surveys, medical histories often rely on personal reporting and subjective recall and result in measurement errors when people convert alcohol consumption to standard units. ${ }^{5}$ Furthermore, relating health outcomes to drinking habits at a single point in time could overlook the effect of historical drinking patterns. ${ }^{5}$ Additionally we only assessed the short-term (3-year) impact of the SoS initiative on ALD outcome. The natural history of this chronic progressive disease might be more accurately observed with a longer follow-up period. However, our year-by-year analysis (Fig 2) did not seem to elucidate a temporal pattern.

Finally, there were important changes in service delivery during the study period that could have impacted our results. Since 2014, all patients admitted with liver disease were assessed by a gastroenterologist/hepatologist within 24 hours, which was not the case previously. Also, in 2016, consistent with national trends in reduction in addiction services, we lost our dedicated hospital alcohol nurses as well as inpatient beds at a local psychiatric facility.

In summary, our study shows that while regulating alcohol sales can modify drinking behaviour, its impact on the clinical spectrum of ALD remains controversial. A complex condition like ALD is likely to need a multifaceted intervention. 


\section{Supplementary material}

Additional supplementary material may be found in the online version of this article at www.rcpjournals.org/clinmedicine:

S1 - Baseline demographic and clinical data in the whole cohort and stratified by admission in phase 1 and 2 .

S2 - Univariate analysis of baseline demographic and clinical variables associated with inpatient mortality following index hospitalisation with decompensated ALD.

\section{References}

1 Williams R, Alexander G, Aspinall R et al. Gathering momentum for the way ahead: fifth report of the Lancet Standing Commission on Liver Disease in the UK. Lancet 2018;392:2398-412.

2 Burton R, Henn C, Lavoie D et al. The public health burden of alcohol and the effectiveness and cost-effectiveness of alcohol control policies: An evidence review. London: Public Health England, 2016.

3 Office for National Statistics. Census 2011: Coastal communities data. ONS, 2014. https://webarchive.nationalarchives.gov. uk/20160130200905/http://www.ons.gov.uk/ons/publications/rereference-tables.html?edition $=$ tcm \% 3A77-382072 [Accessed 25 July 2019].

4 Public Health England. Local alcohol profiles for England. PHE. https://fingertips.phe.org.uk/profile/local-alcohol-profiles [Accessed 25 April 2019].

5 Burton R, Henn C, Lavoie D et al. A rapid evidence review of the effectiveness and cost-effectiveness of alcohol control policies: an English perspective. Lancet 2017;389:1558-80.

6 World Health Organization. International classification of diseases, 10th revision. WHO, 2015.

7 Charif I, Saada K, Mellouki I et al. Predictors of intra-hospital mortality in patients with cirrhosis. Open J Gastroenterol 2001;4:141-8.

8 Crabb DW, Im GY, Szabo G, Mellinger JL, Lucey M. Diagnosis and treatment of alcohol-related liver diseases: 2019 practice guidance from the American Association for the Study of Liver Diseases. Hepatol 2020;71:306-33.

9 Kamath PS, Wiesner RH, Malinchoc M et al. A model to predict survival in patients with end-stage liver disease. Hepatol 2001;33:464-70.

10 Barber KM, Pioli S, Blackwell JE et al. Development of a UK score for patients with end-stage liver disease. Hepatol 2007;46:611A.

11 Department of Health. UK chief medical officers' low risk drinking guidelines. DoH, 2016. https://assets.publishing.service.gov.uk/ government/uploads/system/uploads/attachment_data/ file/545937/UK_CMOs__report.pdf [Accessed 25 July 2019].

12 Lynsdale D (Sensible on Strength initiative responsible office at Brighton \& Hove City Council). Personal correspondence. Unpublished data.

13 Sharma A, Sinha K, Vandenberg B. Pricing as a means of controlling alcohol consumption. 2017. Br Med Bull 2017:123:149-58.

14 Gruenewald PJ, Ponicki WR. The relationship of alcohol sales to cirrhosis mortality. J Stud Alcohol 1995;56:635-41.

15 Stokkeland K, Brandt L, Ekbom A, Ösby U, Hultcrantz R. Morbidity and mortality in liver diseases in Sweden 1969-2001 in relation to alcohol consumption. Scand J Gastroenterol 2006;41:459-64.

16 Tyrfingsson T, Olafsson S, Bjornsson E S, Rafnsson V. Alcohol consumption and liver cirrhosis mortality after lifting ban on bee sales in country with state alcohol monopoly. Eur J Public Health 2015;25:729-31.

17 Guy J, Peters M. Liver disease in women: The influence of gender on epidemiology, natural history, and patient outcomes. Gastroenterol Hepatol 2013;9:633-9.

Address for correspondence: Dr Yazan Haddadin, Brighton and Sussex University Hospitals NHS Trust, Royal Sussex County Hospital, Eastern Road, Brighton BN2 5BE, UK. Email: yazan.haddadin@nhs.net 